\title{
Modeling of the Flow Comparator Prototype as New Primary Standard for High Pressure Natural Gas Flow Metering
}

\author{
Sukhwinder Singh $^{1} \quad$ Gerhard Schmitz $^{1} \quad$ Bodo Mickan $^{2}$ \\ ${ }^{1}$ Institute for Engineering Thermodynamics, Hamburg University of Technology, Germany, \\ \{sukhwinder.singh, schmitz\}@tuhh. de \\ ${ }^{2}$ Physikalisch-Technische Bundesanstalt, Braunschweig, Germany, bodo.mickan@ptb. de
}

\begin{abstract}
The German national metrological institute, PhysikalischTechnische Bundesanstalt, is developing a new concept for volumetric primary standard to calibrate high pressure gas flow meters. The TUHH is supporting these R\&D activities with its competence to elaborate computational models for detailed analysis of complex mechanical systems including fluid flow aspects. The new primary standard is based on a actively driven piston prover to measure the gas flow rate using the time the piston needs to displace a defined enclosed volume of gas in a cylinder.

A computational model written in Modelica ${ }^{\circledR}$ is developed to investigate the Flow Comparator's dynamic behavior. Validation of the model shows good compliance of the piston velocity and differential pressure at the piston in the model with measured data. With this model the control voltage trajectory can be optimized to increase the available measuring time and it allows to gather detailed information about pressure and temperature development at arbitrary chosen locations in the system with high time resolution.
\end{abstract}

Keywords: modeling of multi-domain physical systems, flow comparator, high pressure natural gas flow metering, linear motor, optimization

\section{Introduction}

For the trade with natural gas the uncertainty of high pressure natural gas flow meters is of major importance. The calibration of the flow meters is done with transfer standards which are calibrated by the German national primary standard for high pressure natural gas flow. The current primary standard is a High Pressure Piston Prover (HPPP) (Schmitz and Aschenbrenner; PTB, 1991, 2009). It is owned and operated by the National Metrology Institute of Germany Physikalisch-Technische Bundesanstalt (PTB) and installed on the calibration facility for gas meters pigsar ${ }^{\mathrm{TM}}$ in Dorsten, Germany. The HPPP can be operated with inlet pressures up to 90 bar and flow rates up to $480 \mathrm{~m}^{3} / \mathrm{h}$ (PTB, 1991).

Due to the increasing size and flow rates of the gas flow meters and the limited operation range of the current national standard, a new concept for calibrating gas flow meters is being developed, the Flow Comparator. A develop- ment prototype of the Flow Comparator is used for preliminary tests such as investigating the controllability and the usable flow rate at ambient conditions. A picture of the prototype is shown in Figure 1.

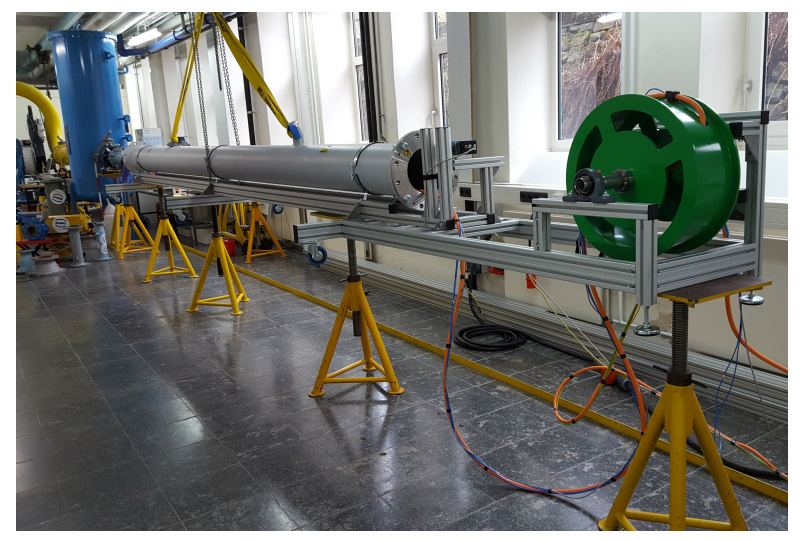

Figure 1. Picture of the Flow Comparator prototype

\section{Experimental Setup}

The key element of the Flow Comparator is a piston in a cylinder. Together they act as an asynchronous linear motor. For this, the cylinder has two layers, one with magnetic properties and the other one acts as an electrical conductor. The stator core with its windings is integrated into the piston. For the electrical power of the stator core a supply cable is connected to the piston. The velocity of the piston is controlled by using a frequency inverter to set the control voltage and frequency for the stator core.

The experimental setup is shown in Figure 2. The differential pressure over the piston is measured with a sensor in the piston. A specified leakage in the piston with a flow sensor measures the fluid flowing through it. With the two sensors, it is possible to compare the piston movement relative to the fluid flow. The piston has an integrated check valve to limit the pressure drop downstream of the piston.

The position of the piston is measured using a distance measuring equipment (DME). The ambient temperature and pressure as well as the temperature and pressure downstream of the cylinder are measured.

A Turbine Meter (TM) is used as transfer standard. The TM measures the volume flow rate using the rotational 
DME $\quad \Delta p, v \quad$ Piston Prover

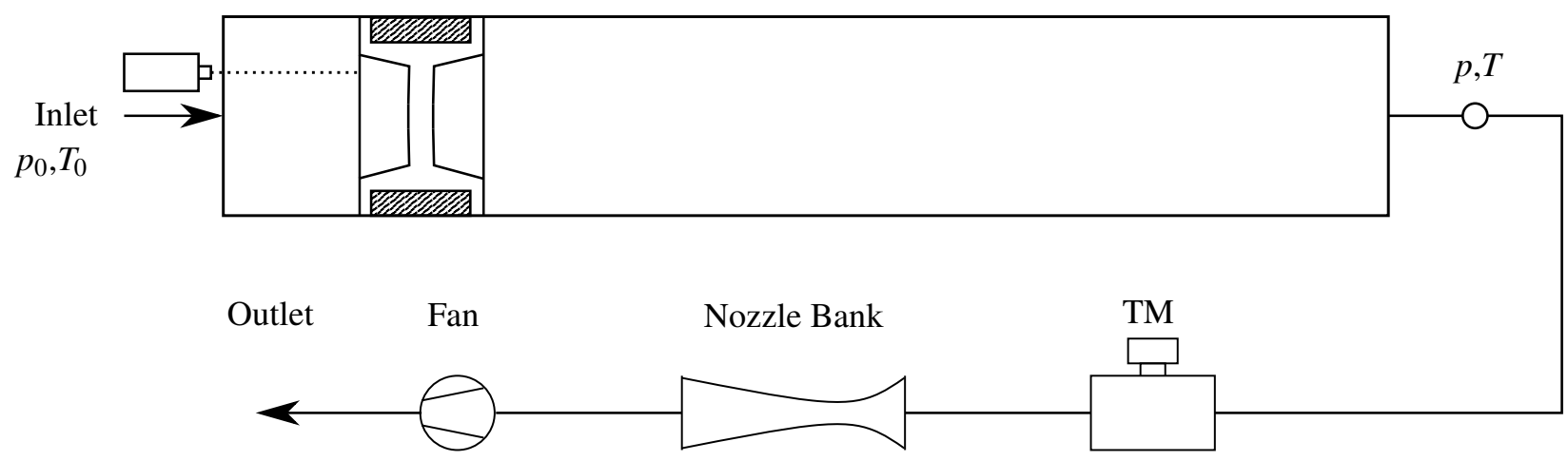

Figure 2. Scheme of the experimental setup with the Flow Comparator prototype

velocity of a turbine inserted into the fluid flow. The rotational speed is measured using a magnetically induced discrete signal of the turbine blades. The nozzle bank is used to set the flow rate and consists of calibrated nozzles with different volume flow rates in a parallel setup. The nozzle bank is not needed for the operation of the Flow Comparator, but provides the advantage of decoupling the experimental setup from pressure fluctuations created by the fan. The fan ensures that the pressure downstream of the nozzle bank is low enough to have a critical flow in the nozzles.

At the beginning of a measurement, the volume flow rate is set by the nozzle bank and the piston moves slowly upstream. At the starting point, the piston is accelerated downstream until the piston velocity is the same as the fluid velocity. The actual measurement phase starts when the piston reaches the defined velocity and moves past a certain point. The measurement phase ends at a defined point downstream where the piston is stopped. The volume flow rate can be calculated as stated in Equation 1, with the volume in between the starting and end point and the time span $\Delta_{F C} t$. Therefore, the Flow Comparator is traceable to the standards of length and time.

$$
\dot{V}_{\mathrm{FC}}=\frac{V_{\mathrm{FC}}}{\Delta_{\mathrm{FC}} t}
$$

In the same time span, the discrete pulses of the turbine meter are counted. The volume flow rate $V_{\mathrm{TM}}$ indicated by the turbine meter can be calculated using a relationship between volume flow rate and indicated signals per time period for the turbine, which is known from previous calibration (or from manufacturer specifications).

The calibration result is the relative deviation $f$ at a certain volume flow rate and pressure. The relative deviation is calculated as stated in equation 2 with the corrected volume flow rate as indicated by the turbine and the corrected volume flow rate as indicated by the Flow Comparator.

$$
f=\frac{\dot{V}_{\mathrm{TM}}^{\mathrm{c}}-\dot{V}_{\mathrm{FC}}^{\mathrm{c}}}{\dot{V}_{\mathrm{FC}}^{\mathrm{c}}}
$$

To improve the calibration accuracy, some corrections are applied to the indicated volume flow rate by the turbine meter and the Flow Comparator. These are explained in the following:

1. With equation 3 it is possible to correct the error caused by the discrete nature of the turbine meter signals. As stated before, the time span $\Delta_{\mathrm{FC}} t$ is the duration of the measurement phase. The time span $\Delta_{\mathrm{TM}} t$ is the duration from the first signal of the turbine meter after the start of the measurement phase to the first signal of the turbine meter after the end of the measurement phase.

$$
\dot{V}_{\mathrm{TM}}^{\mathrm{c}}=\dot{V}_{\mathrm{TM}} \frac{\Delta_{\mathrm{FC}} t}{\Delta_{\mathrm{TM}} t}
$$

2. The following two corrections are applied due to small differences of piston velocity to the fluid velocity. With the differential pressure sensor and the fluid flow velocity sensor it is possible to compare the flow upstream and downstream of the piston.

A non-zero differential pressure at the piston results in a leakage around the piston. The relationship between leakage and differential pressure is stated in Equation 4.

$$
\dot{V}_{\text {leak }, \Delta \mathrm{p}}=a \sqrt{\Delta p}+b
$$

The coefficients $a$ and $b$ can be estimated by experiments.

The volume flow correction with the differential pressure sensor is practical for relatively high leakage flows. For small leakages at the piston the fluid flow velocity sensor can be used. A non-zero velocity indicated by the fluid flow velocity sensor results in a leakage around the piston. The relationship is shown in equation 5 where the coefficients $c$ and $d$ are also experimentally determined.

$$
\dot{V}_{\text {leak }, \mathrm{v}}=c v^{2}+d v
$$




\section{Description of the model}

Modelica ${ }^{\circledR}$ was used as modeling language to describe the physical and dynamic behavior of the Flow Comparator. As simulation environment Dymola is used. A graphical representation of the developed model is shown in Figure 3.

The assumptions used in the model are (von der Heyde et al., 2015):

- pressure losses are proportional to the dynamic pressure,

- the gas flow is one dimensional,

- the system is adiabatic,

- potential energy of the gas is neglected,

- the heat transfer in the gas can be neglected in comparison to convective energy transport.

The air used in the Flow Comparator is sucked out of the experimental hall. Therefore, a constant ambient temperature and pressure can be assumed. This is modeled using a supply volume of infinite size from the Modelica Standard Library (MSL). These boundary conditions are set by equation 6 and equation 7. $p_{\text {In }}$ is the inlet pressure and $T_{\text {In }}$ is the inlet temperature

$$
\begin{aligned}
& p_{\text {In }}=\text { const } . \\
& T_{\text {In }}=\text { const } .
\end{aligned}
$$

The air properties are calculated using an air model of the MSL.

Another boundary condition is set by the nozzle bank. As aforementioned the fan ensures that the pressure downstream of the nozzle bank is low enough to have critical flow in the nozzle. The critical volume flow rate $\dot{V}_{\mathrm{N}}$ in the nozzle is set by Equation 8.

$$
\dot{V}_{\mathrm{N}}=\text { const } \text {. }
$$

The physical behavior of several nozzles is the same to one larger nozzle with equivalent diameter. Therefore, the nozzle bank is modeled as one nozzle with larger diameter based on equations from International Standard DIN EN ISO 9300 (International Organization for Standardization, 2005). The mass flow rate in the nozzle is calculated in Equation 9 using the critical volume flow rate $\dot{V}_{\mathrm{N}}$ and the upstream density $\rho$.

$$
\dot{m}_{\mathrm{N}}=\dot{V}_{\mathrm{N}} \rho
$$

For the model, the measuring cylinder is divided into one volume upstream of the piston and one volume downstream of the piston. The enclosed gas volumes depend on the position of the piston and change volume with piston movement. They can store mass $m$, internal energy $m u$ and momentum $m v$ as described in Equation 10, 11 and 12.

$$
\begin{gathered}
\frac{d m}{d t}=\dot{m}_{i}+\dot{m}_{i+1} \\
\frac{d}{d t} m u=\dot{m}_{i}\left(h_{i}+\frac{v_{i}^{2}}{2}\right)+\dot{m}_{i+1}\left(h_{i+1}+\frac{v_{i+1}^{2}}{2}\right) \\
+\left(\frac{p_{i+1}-p_{i}+p_{\mathrm{f}, i+1}-p_{\mathrm{f}, i}}{2}\right) \dot{V}_{i}+\dot{Q} \\
\frac{d}{d t} m v=\dot{m}_{i}\left|v_{i}\right|+\dot{m}_{i+1}\left|v_{i+1}\right|-A\left(p_{i+1}-p_{i}\right) \\
-A\left(p_{\mathrm{f}, i+1}-p_{\mathrm{f}, i}\right)
\end{gathered}
$$

In direction of fluid flow a spatial discretization is applied which leads to a number of finite volumes in the enclosed gas volume. For the discretization the finite volume method with a staggered grid approach is used. Figure 4 shows the placement of variables on a 1D staggered mesh. The scalar variables (e.g. pressure, density etc.) are located in the control volume cell center while the velocity and momentum variables are stored on the cell faces.

Equations 10-12 are applied for each finite volume in the enclosed gas volume. $\dot{m}$ is the mass flow rate, $h$ the specific enthalpy, $v$ the mean velocity in the cross area, $p$ the static pressure, $p_{\mathrm{f}}$ the pressure loss due to friction, $V$ the volume and $\dot{Q}$ is the heat flow.

The pressure loss is calculated using the detailed characteristic wall friction model from the MSL. The model calculates the pipe friction coefficient depending on the Reynolds number and the relative roughness. A heat port is included in the model of the enclosed gas volume and can be connected to another heat port, e.g. the ambient or the piston. The heat flow in the model is calculated using a heat transfer model from the MSL.

The position and motion of the piston is determined by the equation of motion as stated in Equation 13.

$$
m_{\mathrm{P}} \ddot{S}_{\mathrm{P}}=p_{1} A_{\mathrm{P}}-p_{2} A_{\mathrm{P}}-F_{\mathrm{R}, \mathrm{P}}-F_{\mathrm{R}, \mathrm{C}}+F_{\mathrm{LM}}
$$

$p_{1}$ and $p_{2}$ are the pressures of the fluid upstream and downstream of the piston, $F_{\mathrm{R}, \mathrm{P}}$ is the roll resistance of the piston, $F_{\mathrm{R}, \mathrm{C}}$ the resistance of the connection cable and $F_{\mathrm{LM}}$ is the force of the linear motor to drive the piston. The roll resistance of the piston is modeled using a constant rolling resistance coefficient as stated in equation 14 with $c_{\mathrm{R}}$ being the roll resistance coefficient and $F_{\mathrm{N}}$ being the normal force of the piston.

$$
F_{\mathrm{R}, \mathrm{P}}=c_{\mathrm{R}} F_{\mathrm{N}}
$$

The resistance due to the weight of the connection cable $F_{\mathrm{R}, \mathrm{C}}$ is modeled as shown in Equation 15 with $\mathrm{g}$ being the gravitational force, $m_{\mathrm{C}}$ the total weight of the connection cable, $s$ the current position of the piston and $l$ the total length of the connection cable. 


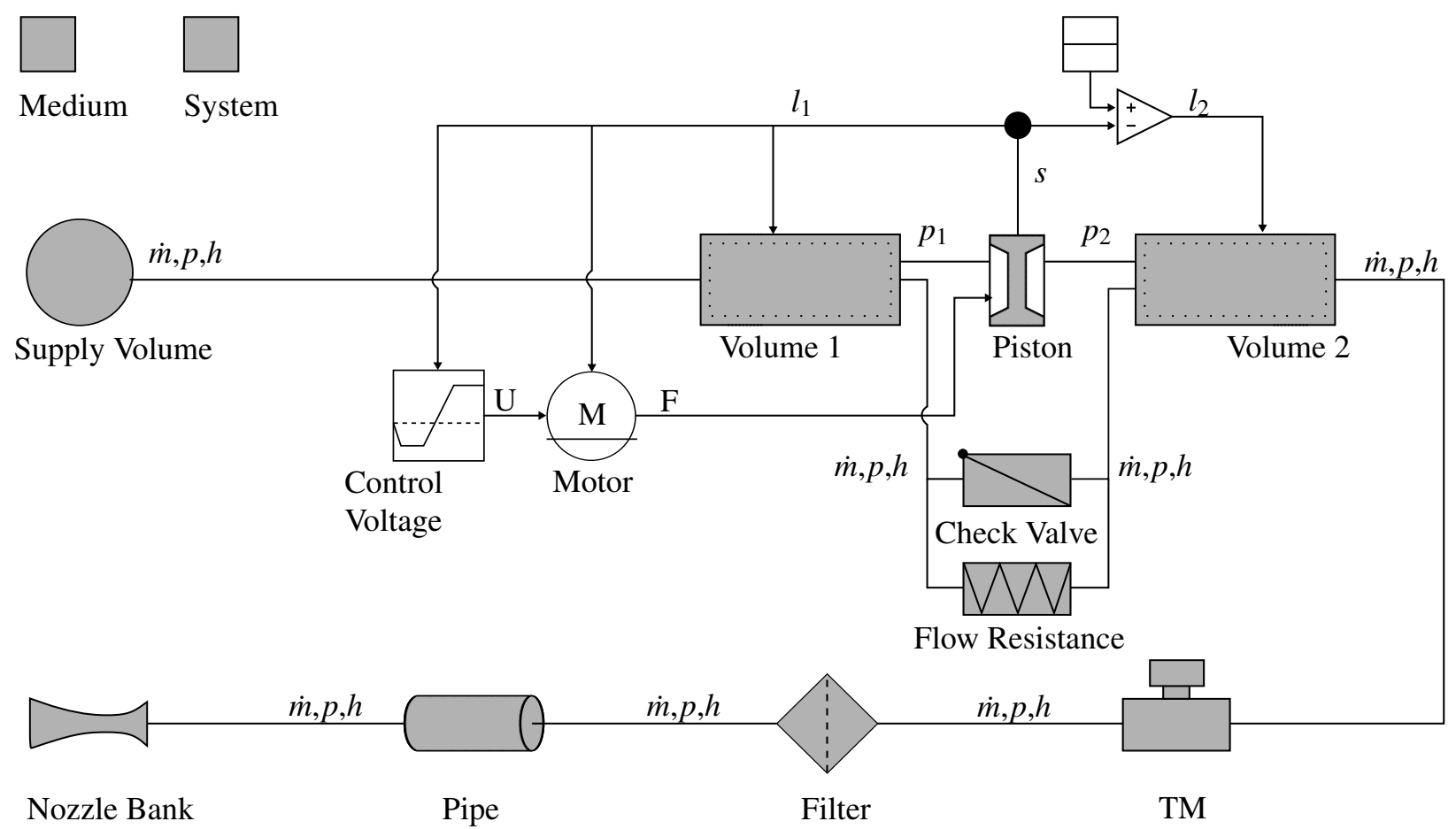

Figure 3. Graphical representation of the computational model

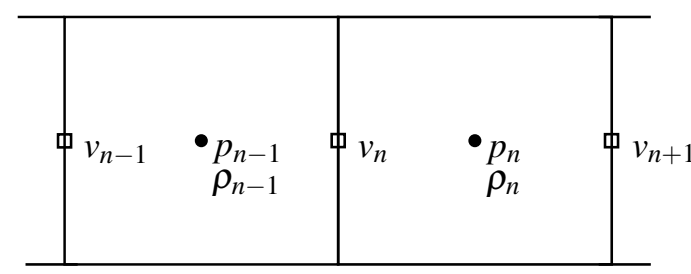

Figure 4. Placement of variables using the finite volume method with a staggered grid approach

$$
F_{\mathrm{R}, \mathrm{C}}=g \cdot m_{\mathrm{C}} \frac{s}{l}
$$

The movement of the piston can be controlled with the linear motor integrated into the piston. As a first approach to model the force of the linear motor, a function depending on control voltage input and velocity of the piston is used. The function is derived by measuring the velocity of the piston for several control voltages and different flow resistances. The fitting function used is shown in Equation 16.

$$
F_{\mathrm{LM}}=\alpha\left(I-I_{\mathrm{S}}\right) \cdot\left(\frac{v}{U_{\text {control }}}-v_{\mathrm{S}}\right)+F_{\mathrm{F}}
$$

$\alpha$ is a proportional constant, $I$ is the electric current of the linear motor, $I_{\mathrm{S}}$ is the magnetizing current for the magnetic field, $v$ is the velocity of the piston, $v_{\mathrm{S}}$ is the normalized synchronous velocity of the linear motor and $F_{\mathrm{F}}$ is the friction force of the piston. The experimental data and the result of the fitting is shown in Figure 5 where each line represents a different control voltage. The experimental data and fitting function are close-fitting overall. The output of the linear motor model is the force accelerating the piston. The motor model needs the current velocity of the piston and control voltage of the frequency inverter as input.

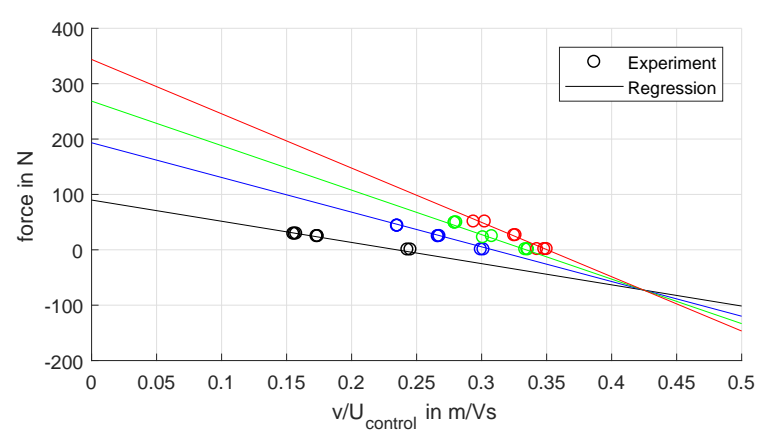

Figure 5. Experimental results and fitting function for the forcevelocity relation of the linear motor

The control voltage model depends on the position of the piston. Initially, it increases linearly to a predefined negative value. When the starting position of the piston is reached, the control voltage increases to a defined positive value.

The check valve in the piston is modeled as a check valve between the gas volumes of the measuring cylinder. It opens at a specific differential pressure and enables a fluid flow from volume 1 to volume 2 . The check valve is modeled with a hysteresis to avoid chattering. The mass flow rate through the check valve is proportional to the pressure drop over the check valve. For this a pressure loss coefficient $\zeta_{\mathrm{CV}}$ as specified by the manufacturer is used.

The flow resistance model describes the leakage between measuring cylinder and piston as there is a small 
diameter difference between the two. To describe the relation of mass flow rate and pressure drop a function is derived from experiments.

The turbine meter model uses a constant pressure loss coefficient $\zeta_{\mathrm{TM}}$ to model the pressure loss in the turbine meter as shown in Equation 17. $\rho$ is the density and $v_{A}$ the mean velocity in the cross area $A$ of the turbine meter.

$$
\Delta p=\zeta_{\mathrm{TM}} \frac{\rho}{2} v_{\mathrm{A}}^{2}
$$

The pressure loss coefficient $\zeta_{\mathrm{TM}}$ is taken from measurement data. The relationship between indicated volume flow rate and real volume flow rate of the turbine is modeled as stated in Equation 18 which is a further development of the equation described in (Mickan et al., 2010). $v_{i, \text { rel }}$ is the relative indicated volume flow rate in relation to the maximal possible volume flow rate for the specific turbine meter, $v$ the real volume flow rate, $\rho$ is the density and $v_{\mathrm{i}}$ the indicated volume flow rate. The coefficients $a$, $b, A$ and $B$ are results of different experiments.

$$
\dot{v}_{\mathrm{i}, \text { rel }}-\left(a+b v_{\mathrm{i}, \mathrm{rel}}\right)=A \rho v^{2}-B \rho v v_{\mathrm{i}}
$$

The filter is modeled as simple flow resistance and the pressure loss is determined as shown in equation 17. The DynamicPipe model of the MSL is used as the pipe model. It uses the balance equations for mass $m$, internal energy $m u$ and momentum $m v$ shown in Equation 19, 20 and 21 on a number of finite volumes in the pipe. $\dot{m}$ is the mass flow, $h$ the specific enthalpy, $v$ the velocity, $A$ the cross-sectional area of the pipe, $p$ the pressure and $F_{\mathrm{F}}$ the friction force in the pipe (Mickan et al., 2010).

$$
\begin{gathered}
\frac{d m}{d t}=\dot{m}_{i}+\dot{m}_{i+1} \\
\frac{d}{d t} m u=\dot{m}_{i} h_{i}+\dot{m}_{i+1} h_{i+1} \\
+\frac{1}{2}\left(v A\left(p_{i+1}+p_{i}\right)+v F_{\mathrm{F}}\right) \\
\frac{d}{d t} m v=\dot{m}_{i}\left|v_{i}\right|+\dot{m}_{i+1}\left|v_{i+1}\right|-A\left(p_{i+1}-p_{i}\right)-F_{\mathrm{F}}
\end{gathered}
$$

\section{Verification}

The verification of a model shows the correct physical implementation of a model and the accurate solution of the equation system. For this, different parameter of the model are varied and piston velocity and pressure difference over the piston are used as measure for verification.

For the solution of the equation system, the solver Dassl with a relative tolerance of $10^{-6}$ is used. A further decrease in relative tolerance as well as using other solvers does not change the model trajectory. For the volumes of the measuring pipe, 8 discrete volumes are used and for the pipe, 4 discrete volumes are used.
In Figure 6, the piston velocity for different control voltages of the frequency inverter is shown. The acceleration of the piston is the same as it primarily depends on the power ramp of the frequency inverter. Higher control voltages have a greater maximum peak velocity as well as end velocity.

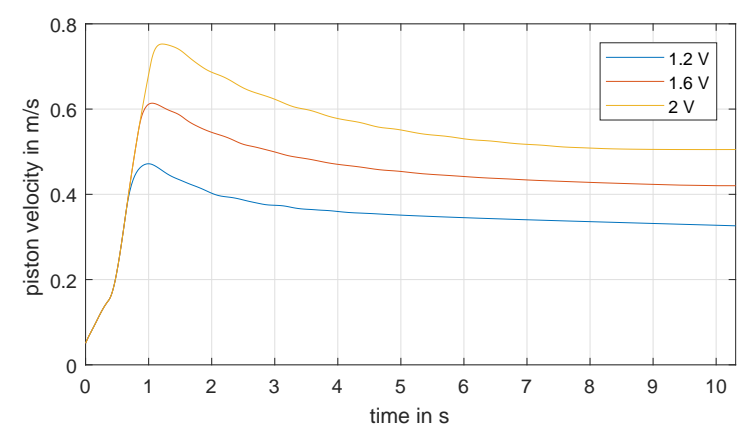

Figure 6. Piston velocity over time for different frequency inverter control voltages

The piston velocity for different rising time for the ramp of the frequency inverter is shown in Figure 7. As expected, a lower rising time results in a higher acceleration of the piston due to the faster increasing current in the linear motor and therefore a higher induced force on the piston. After the control voltage has increased to the defined value, the difference in piston velocity decreases and the piston velocity is almost the same at the end of the simulation.

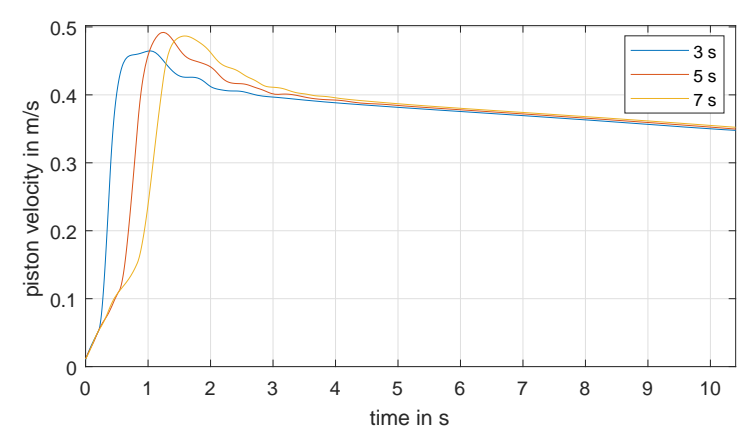

Figure 7. Piston velocity over time for different frequency inverter rising time

In Figure 8, the differential pressure at the piston for different volume flow rates of incoming air flow is shown. For a volume flow rate of $65 \mathrm{~m}^{3} / \mathrm{h}$, the piston velocity is close to the velocity of the air flow and the differential pressure is almost zero after the acceleration process. As the piston velocity is almost independent of the air flow rate, a lower volume flow rate results in a negative differential pressure at the piston. A negative differential pressure means that the downstream pressure is higher than the upstream pressure due to the faster movement of the piston in comparison to the air flow. Accordingly, a higher air flow rate results in a positive differential pressure around the piston. 


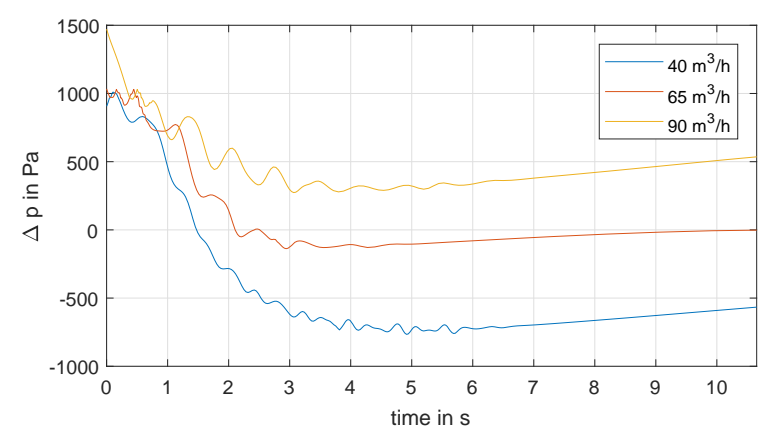

Figure 8. Differential pressure at the piston over time for different air flow rates

\section{Validation}

To use the model for further predictions of the dynamic behavior of the Flow Comparator, the accuracy of the model is highly relevant. The accuracy is affected by the aforementioned mentioned general assumptions, the accuracy of the empirical correlation for the linear motor and frequency inverter, the assumptions for the friction force, and further simplifications.

For the model's validation, the prototype's measurement data is used. The experiments are conducted as described in Section 2. The position of the piston is measured using the laser distance measuring equipment.

The simulations are carried out with the same control voltage for the frequency inverter as the experiment for a given air flow rate to validate the empirical approach used for frequency inverter and linear motor. The moment when the piston starts moving forward is set as $t=0 \mathrm{~s}$ and the validation is only done for that part of the experiment as this is the important part of the measurement.

In Figure 9, the piston velocity in simulation and experiment is shown over time for a volume flow rate of $116 \mathrm{~m}^{3} / \mathrm{h}$ and a control voltage of $1.95 \mathrm{~V}$. In the first $0.5 \mathrm{~s}$, the linear motor is not active due to the rising ramp of the frequency inverter and the piston is accelerated by the differential pressure at the piston. In this time frame, the simulation shows a lower acceleration for the piston than in the measurement. When the linear motor is active, the simulated piston acceleration is higher than the measured acceleration of the piston. In simulation and measurement, the maximum piston velocity is similar. The decrease in piston velocity due to the increasing resistance force of the connection cable shows a similar behavior in simulation and measurement. Overall, a relatively good accordance of the simulated and measured piston velocity for a volume flow rate of $116 \mathrm{~m}^{3} / \mathrm{h}$ is achieved.

The differential pressure at the piston over the time for the simulation and the measurement is shown in Figure 10 for a volume flow rate of $116 \mathrm{~m}^{3} / \mathrm{h}$ and a control voltage of $1.95 \mathrm{~V}$. The differential pressure around the piston between simulation and measurement shows a time offset. The simulation has an immediate differential pressure drop when the piston accelerates while the measurement

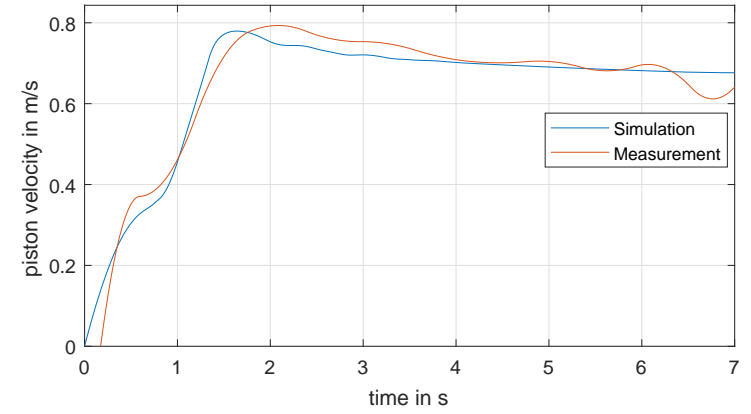

Figure 9. Comparison of the piston velocity over the time in the model and the measured data for a volume flow rate of $116 \mathrm{~m}^{3} / \mathrm{h}$ and a control voltage of $1.95 \mathrm{~V}$

data shows a delayed decrease in differential pressure. The slope of differential pressure decrease is the same for simulation and measurement. Except for the time offset, the simulation and measurement show a good agreement in behavior of the differential pressure at the piston.

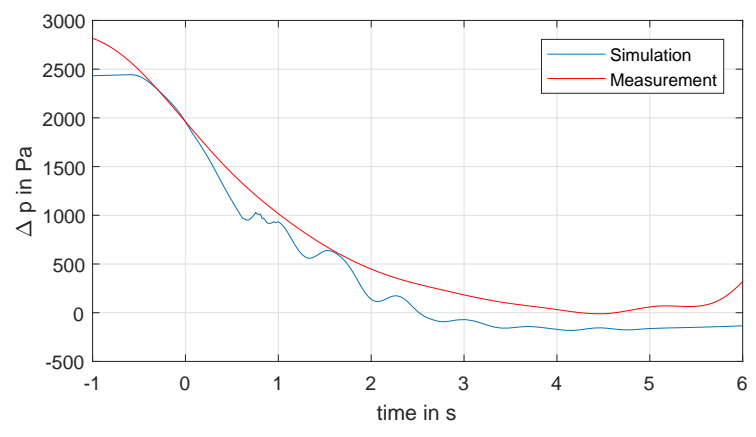

Figure 10. Comparison of the differential pressure at the piston over the time in the model and the measured data with a time offset $t_{\text {off }}=-1.9 \mathrm{~s}$ for a volume flow rate of $116 \mathrm{~m}^{3} / \mathrm{h}$ and a control voltage of $1.95 \mathrm{~V}$

For validation of lower volume flow rates, the simulation and measurement data for $V=65 \mathrm{~m}^{3} / \mathrm{h}$ is shown in Figure 11. The simulation again has a lower piston acceleration at the beginning and a higher piston acceleration when the linear motor is active. After reaching the maximum piston velocity simulation and experimental data have an similar decrease in piston velocity. Therefore, a good accordance between measurement and simulation is also achieved for lower volume flow rates.

The difference in acceleration between simulation and measurement data due to the air flow is caused partly by the modeling of the pistons resistance force and the approach of using a constant roll resistance coefficient. This may lead to the described difference. The accuracy of the linear motor model may be increased by using an empirical approach which describes the dynamic part of the acceleration in more detail. 


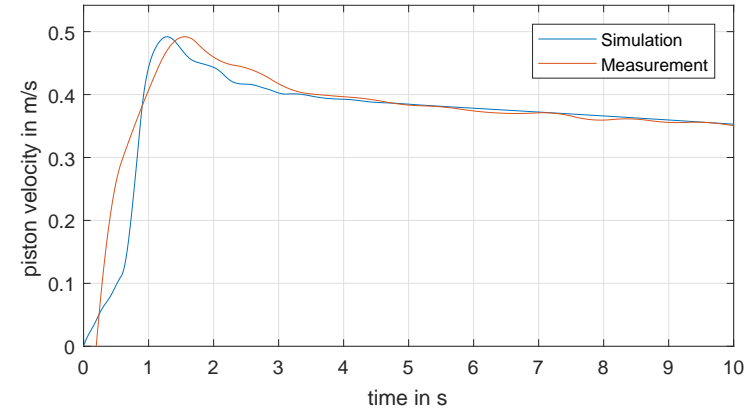

Figure 11. Comparison of the piston velocity over the time in the model and the measured data for a volume flow rate of $65 \mathrm{~m}^{3} / \mathrm{h}$ and a control voltage of $1.25 \mathrm{~V}$

\section{Optimized control voltage trajec- tory}

The model is used to optimize the control voltage trajectory of the frequency inverter to increase the available measuring time. The differential pressure at the piston is used as measure for the available measuring time.

There are different aspects to consider when optimizing the control voltage. The pressure drop at the piston results in a lower density downstream of the piston and accordingly less mass in the measuring cylinder. In order to increase the pressure and density downstream of the piston an overshoot in piston velocity is necessary. Accordingly, an overshoot in control voltage needs to be applied. The piston's resistance increases while it moves downstream due to the connection cable. Therefore, an increase in driving force is necessary.

In Figure 12 the trajectory of the optimized and nonoptimized control voltage over time for a volume flow rate of $116 \mathrm{~m}^{3} / \mathrm{h}$ is shown. The control voltage in the optimized case has an maximum value of $2.8 \mathrm{~V}$ and thus has an overshoot of $0.85 \mathrm{~V}$. It decreases to a value close to the non-optimized control voltage and then increases with a constant slope.

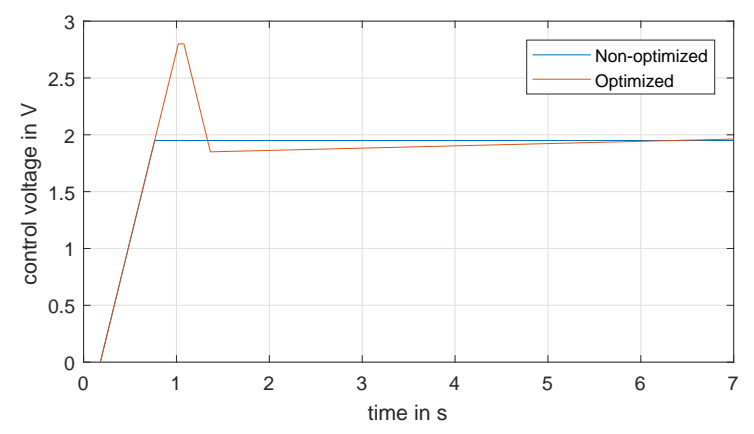

Figure 12. Comparison of the optimized and non-optimized frequency inverter control voltage over time for a volume flow rate of $116 \mathrm{~m}^{3} / \mathrm{h}$

The piston velocity over time is shown in Figure 13 for both regarded cases. The piston velocity is the same as long as the control voltage is increasing and has the same value. At the control voltage overshoot the piston velocity for the optimized case overshoots, too. After the overshoot the piston velocity in the optimized case reaches the air flow velocity much earlier than in the non-optimized case. Furthermore, the piston velocity in the optimized case stays close to the flow velocity while the piston velocity in the non-optimized case decreases continuously.

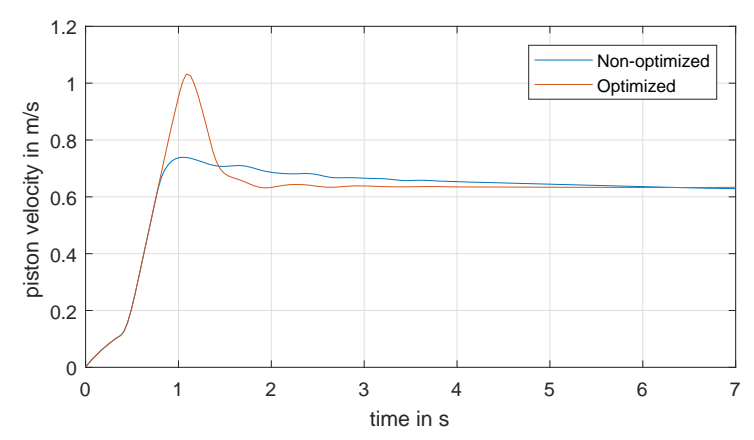

Figure 13. Comparison of the piston velocity over time for the optimized and non-optimized frequency inverter control voltage for a volume flow rate of $116 \mathrm{~m}^{3} / \mathrm{h}$

The differential pressure at the piston over time for the non-optimized and optimized case is shown in Figure 14. At the beginning of the measurement the differential pressure at the piston is the same for both regarded cases. The differential pressure at the piston decreases faster to the desired value of $\Delta p=0 \mathrm{~Pa}$ than in the non-optimized case. Furthermore, in the optimized case the differential pressure stays in a close range around $\Delta p=0 \mathrm{~Pa}$ during the measurement. In comparison, the differential pressure at the piston in the non-optimized case is increasing.

The maximum permitted differential pressure at the piston during the measuring time is set to $\Delta p=50 \mathrm{~Pa}$. With this restriction the measuring time of the optimized case is $4.5 \mathrm{~s}$ long while in the non-optimized case the measuring time is about $2.5 \mathrm{~s}$. The measuring time can be increased by $80 \%$ using the optimized control voltage trajectory.

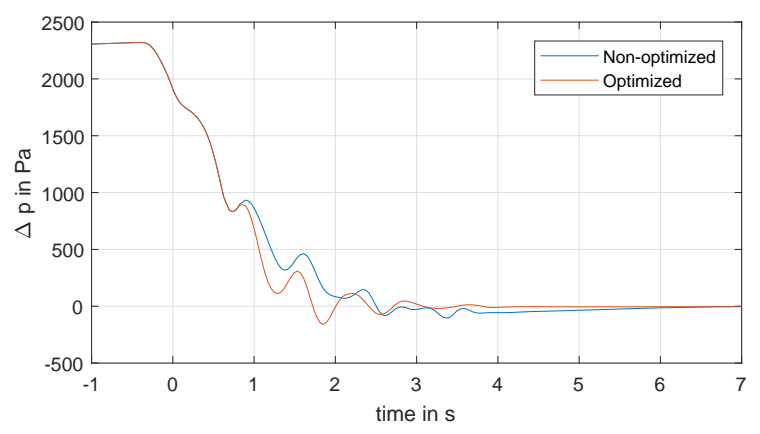

Figure 14. Comparison of the differential pressure at the piston over time for the optimized and non-optimized frequency inverter control voltage for a volume flow rate of $116 \mathrm{~m}^{3} / \mathrm{h}$ 


\section{Conclusion and Outlook}

A model of the Flow Comparator is implemented in Modelica ${ }^{\circledR}$. The model is successfully verified and validated against measurement data. With the model the frequency inverter control voltage trajectory is optimized to maximize the available measuring time. With this simple optimization, the measuring time could be increased by $80 \%$ in the model. This result of optimization will allow to extend the upper limits of flow rate usable for calibrations. Furthermore, the possibility to gather detailed information about pressure and temperature development at arbitrary chosen locations in the system with high time resolution enables much better and more reliable statements about the accuracy of flow rate measurement with this system.

The model uses an empirical approach to model the linear motor's force. In future work, the linear motor should be modeled using physically based equations. Additionally, it will be essential to extend the model by heat transfer from the motor components to the gas to complete the modeling of the overall thermodynamic performance of the piston prover.

Furthermore, the optimization of the frequency inverter control voltage should be done using a more detailed approach. For this the position depending resistance as well as the increase of resistance due to the connection cable weight needs to be measured exactly.

In future work the check valve model and the model of the leakage between piston and cylinder can be improved by using a greater number of measurements to describe their behavior.

\section{References}

International Organization for Standardization. DIN EN ISO 9300: Durchflussmessung von Gasen mit Venturidüsen bei kritischer Strömung. 2005.

B. Mickan, R. Kramer, and V. Strunck. Transient response of turbine flow meters during the application at a high pressure piston prover. In 15th Flow Measurement Conference (FLOMEKO). Linköping University Electronic Press, 2010.

Physikalisch-Technische Bundesanstalt PTB. Prüfschein der Rohrprüfstrecke. 1991.

Physikalisch-Technische Bundesanstalt PTB. PTB Mitteilungen. Special Issue Volume 119 no.1, 2009.

G. Schmitz and A. Aschenbrenner. Experience with a Piston Prover as the New Primary Standard of the Federal Republic of Germany in High-Pressure Gas Metering. In Proceedings of the 18th World Gas Conference, Berlin, 8.-12.7.1991.

M. von der Heyde, G. Schmitz, and B. Mickan. Modeling of the German National Standard for High Pressure Natural Gas Flow Metering in Modelica. In Proceedings of the 11th International Modelica Conference, Versailles, France, September 21-23, 2015. Linköping University Electronic Press, sep 2015. doi:10.3384/ecp15118663. URL https://doi. org/10.3384/ecp15118663. 